Methods: Adult $(\geq 18 y 0)$ patients with symptomatic, refractory inflammatory mono- or oligoarthritis were included for RSO with Yttrium-90 citrate as part of a phase-III, prospective, open-label non-controlled trial. All patients were required to have failed 6-months of medical therapy and 2 intraarticular injections and have minimal evidence of cartilage or bone destruction. Only large and medium-sized joints were included (i.e. knees, ankles, wrists and elbows). The dose of Yttrium was adjusted based on the size of joint. Follow-up evaluations were done at 3, 6 and 12 months after RSO. Safety was assessed by patient and clinician reported adverse events. Clinical response was measured by improvement in joint tenderness, effusion and range of motion.

Results: A total of 74 patients and 83 joints ( $88 \%$ knees) were treated with Ytrium-90 citrate. The underlying diagnosis included $25.7 \%$ RA, 34\% $\mathrm{SpA}, 11 \% \mathrm{JIA}$, and $30.3 \%$ other inflammatory arthritis. Complications included 3 post-RSO flares, 1 septic joint and 2 injection site skin infections. Joint tenderness was reported in $93.8 \%$ of joints at baseline, compared to $50.0 \%$ at $3 \mathrm{mo}(\mathrm{p}<0.001), 55.6 \%$ at $6 \mathrm{mo} \quad(\mathrm{p}<0.001)$ and $40.4 \%$ at $12 \mathrm{mo}(p<0.001)$. Joint effusion was present in $95.1 \%$ of joints at baseline, $44.3 \%$ at $3 \mathrm{mo} \quad(p<0.001), 51.4 \%$ at $6 \mathrm{mo} \quad(p<0.001)$ and $47.4 \%$ at $12 \mathrm{mo}(\mathrm{p}<0.001) .73 .9 \%$ of joints had improvement in range of motion at $3 \mathrm{mo}, 55.9 \%$ at $6 \mathrm{mo}$ and $60.7 \%$ at $12 \mathrm{mo}$.

Conclusion: These results confirm the clinical efficacy and safety of Yttrium90-citrate RSO for refractory synovitis with a sustained clinical benefit at 12 months. This is the first such study in a Canadian cohort. RSO with Ytrium is a safe alternative to surgical synovectomy in refractory cases.

Disclosure of Interests: Nadil Zeiadin: None declared, Eric Turcotte: None declared, Emmanouil Rampakakis : None declared, Helena Senta: None declared, John Sampalis Employee of: JSS Medical Research, Carter Thorne Grant/research support from: Investigator-initiated studies: Amgen, Pfizer. RCTs: Abbvie, Celgene, CaREBiodam, Novartis, Pfizer, Consultant for: Advisory board: Abbvie, Amgen, Celgene, Lilly, Medexus/Medac, Merck, Novartis, Pfizer, Sanofi. Consultant: Abbvie, Centocor, Janssen, Lilly, Medexus/Medac, Pfizer, Speakers bureau: Medexus/Medac

DOI: 10.1136/annrheumdis-2019-eular.431

\section{FRI0167 DOES BARICITINIB HAVE A SPECIFIC EFFECT PATIENT'S REPORTED OUTCOME? COMPARISON OF EFFECTS ON PAIN AND PATIENT GLOBAL ASSESSMENT WITH TNF INHIBITORS}

Eiji Torikai ${ }^{1}$, Daisuke Suzuki ${ }^{2} .{ }^{1}$ Iwata city hospital, Iwata, Japan; ${ }^{2}$ Hamamatsu university school of medicine, Hamamatsu, Japan

Background: Baricitinib (bari) is approved for treating moderate-severe rheumatoid arthritis (RA) in many countries, including Japan. Bari is an oral Janus kinase (JAK1)/JAK2 selective inhibitor and has shown good efficacy in RA and an adequate response to conventional synthetic DMARDs in some clinical trials. Pain and patient global assessment (PGA) are common symptoms for patients with RA. Some trials suggest that bari may have a supplementary benefit on patients' reported outcomes (PRO). Our goal was to determine the impact of each treatment on pain and $P G A$.

Objectives: We evaluated and compared the impact of bari and TNF inhibitors (TNFi) on pain and PGA and correlation with PRO and clinical assessments for Japanese RA patients who have an inadequate response to csDMARDs in real world multicenter clinical data.

Methods: We included 60 Japanese RA patients with an inadequate response to csDMARDs. We classified patients into two groups, one treated with bari (B group; 24 females and 8 males) and the other with TNFi (TNFi group; 22 females and 6 males). Patients were scheduled to receive TNFi (GLM $50 \mathrm{mg} /$ month or ETN $50 \mathrm{mg} /$ week) in TNFi group or bari 4 or $2 \mathrm{mg} /$ day in $\mathrm{B}$ group as a monotherapy or in combination with csDMARDs. We evaluated swollen joint counts on 28 joints (SJC), tender joint counts on 28 joints (TJC), visual analog scale of pain (VAS pain) (0-100 mm), PGA (0-100 mm), and Clinical Disease Activity Index (CDAI) at baseline, 4 weeks, and 12 weeks. First, we compared the changes in SJC, TJC, CDAI, VAS pain, and PGA at 4 and 12 weeks after treatments between the two groups. Second, we evaluated the correlation between PROs (VAS pain or PGA) and variations in clinical evaluation items (SJC, TJC, and CDAI).

Results: There were no significant differences in background at baseline between the two groups (Table 1). Baseline SJC, TJC, and CDAI scores; VAS pain; and PGA were $6.67 \pm 3.81,7.93 \pm 3.69,26.35 \pm 8.77,61.2$ \pm 21.8 , and $62.2 \pm 18.4$ in the bari group, respectively, and $6.13 \pm$ $3.21,7.27 \pm 3.56,24.65 \pm 9.72,63.9 \pm 22.7$, and $64.2 \pm 18.4$ in the TNFi group, respectively. SLC, TJC, CDAI scores, VAS pain, and PGA in both groups were significantly improved 1 month after treatment. This tendency continued for 3 months after treatment. There were no significant differences in the improvement rate of SJC, TJC, CDAI scores, VAS pain, and PGA between the two groups at each follow-up time point after treatment. VAS pain scores were significantly correlated with SJC and CDAl scores in both groups (B group; $r=0.651, p<0.01$ and TNFi group; $r=0.688, p<0.01)$. PGA scores were significantly correlated with SJC and CDAl scores in both groups (B group; $r=0.657, p$ $<0.01$ and TNFi group; $r=0.613, p<0.01$ ).

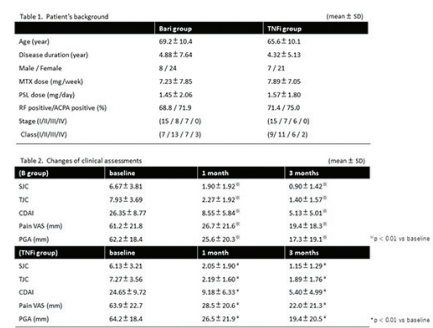

Conclusion: Our results indicate that the effect of bari on pain and PGA is correlated with the improvement rate in disease activity. Bari treatment is not significantly different than TNFi, at least during the short term, in real world clinical data.

\section{REFERENCE:}

[1] Perricone C, et al. Rheumatology (Oxford) 2012;51:866-73

Disclosure of Interests: None declared

DOI: 10.1136/annrheumdis-2019-eular.4515

\section{FRI0168 POSITIVE EFFECTS OF AN ANTI-INFLAMMATORY DIET IN RHEUMATOID ARTHRITIS}

Anna Vadell, Erik Hulander, Linnea Barebring, Inger Gjertsson, Helen Lindqvist, Anna Winkvist. Institute of Medicine, Gothenburg, Sweden

Background: Many patients with rheumatoid arthritis (RA) experience symptom relief of certain foods and earlier research has shown positive effects of food and food components on clinical outcomes of RA. Food components may potentiate each other in their effects on RA, but even so, studies combining foods and food components with probable effects on RA are scarce. Furthermore, comorbidities are common in RA and the risk of cardiovascular disease is elevated in this group. Assumed mechanisms include classical risk factors such as systemic inflammation, dyslipidemia as well as an unfavorable body composition.

Objectives: We investigated if a diet combining foods with anti-inflammatory effects, i.e. an anti-inflammatory portfolio diet, could reduce RA disease activity, markers of inflammation, body composition and blood lipid levels compared to a control diet.

Methods: In this controlled cross-over trial, 50 patients with RA were randomized to start with either intervention or control diet for ten weeks followed by a wash-out period of four months. The intervention diet was an anti-inflammatory portfolio diet containing foods rich in omega-3 fatty acids, fibers and probiotics. The control diet was a typical Swedish diet. Food equivalent to $50 \%$ of daily energy needs was delivered home weekly. For the remaining intake, participants were instructed to consume similar foods as the study diet regimen. Primary outcome measure was change in DAS28, using ESR. Secondary outcomes included changes in blood lipid levels, ESR and CRP as well as body composition.

Results: No significant difference in DAS28 between the groups was seen using Mixed Models analyze $(p=0.116)$. However, DAS28 was significantly lower after the intervention diet compared to after control diet $(p=0.04)$ and a significant reduction over time was seen in the intervention group $(p=0.012)$ using Wilcoxon signed rank test. HDL-cholesterol was increased and TG were lowered after intervention compared to control, analyzed using Mixed Models ( $p=0.046$ and $p=0.006$ respectively). In addition, there was a non-significant trend towards lowered LDL-cholesterol $(p=0.077)$. Comparing after intervention to after control, there were non-significant lower weight, BMI and fat mass after the intervention $(p=0.077, p=0.075$ and $p=0.058$ respectively). None of the other outcomes changed significantly between groups. 
Conclusion: This study showed positive effects on disease activity and blood lipid levels of a diet combining foods with anti-inflammatory properties, but the results must be confirmed in larger studies.

\section{REFERENCES:}

[1] Agca R, et al. EULAR recommendations for cardiovascular disease risk management in patients with rheumatoid arthritis and other forms of inflammatory joint disorders: 2015/2016 update. Annals of the rheumatic diseases. 2017;76(1):17-28.

[2] Piepoli MF, et al. 2016 European Guidelines on cardiovascular disease prevention in clinical practice: The Sixth Joint Task Force of the European Society of Cardiology and Other Societies on Cardiovascular Disease Prevention in Clinical Practice (constituted by representatives of 10 societies and by invited experts)Developed with the special contribution of the European Association for Cardiovascular Prevention \& Rehabilitation (EACPR). European heart journal. 2016;37(29):2315-81.

[3] Sköldstam L, et al. An experimental study of a Mediterranean diet intervention for patients with rheumatoid arthritis. Ann Rheum Dis. 2003;62(3):20814

[4] SLV. [Available from: https://www.livsmedelsverket.se/matvanor-halsamiljo/kostrad-och- matvanor/matvanor-undersokningar/riksmaten-201011-vuxna.

[5] Tedeschi SK, et al. Diet and Rheumatoid Arthritis Symptoms: Survey Results From a Rheumatoid Arthritis Registry. Arthritis Care Res (Hoboken). 2017;69(12):1920-5.

Disclosure of Interests: Anna Vadell: None declared, Erik Hulander: None declared, Linnea Barebring: None declared, Inger Gjertsson: None declared, Helen Lindqvist Employee of: Yes, for Janssen-Cilag $A B$ in 2001-2003, Before I was a phD-student and within a different field (dementia). My research field is within diet and Rheumatology., Anna Winkvist: None declared

DOI: 10.1136/annrheumdis-2019-eular.4522

\begin{tabular}{|l|l}
\hline FRI0169 & DOES INITIATING TOCILIZUMAB LEAD TO BETTER \\
& DISEASE CONTROL COMPARED TO INITIATING MTX \\
& WITH LOW-MODERATE DOSE PREDNISONE IN EARLY \\
& RHEUMATOID ARTHRITIS; AN INDIRECT COMPARISON \\
& OF U-ACT-EARLY AND CAMERA-II TREAT-TO-TARGET \\
& TRIALS
\end{tabular}

Maxime Verhoeven $^{1}$, Janneke Tekstra ${ }^{1}$, M Jacob. van Laar ${ }^{1}$, Johannes Wj Bijlsma ${ }^{1}$, Attila Pethoe-Schramm ${ }^{2}$, Michelle Borm ${ }^{3}$, Floris Lafeber ${ }^{1}$, Paco Welsing ${ }^{1} .{ }^{1}$ University Medical Center Utrecht, Utrecht, Netherlands; ${ }^{2}$ Hoffmann-La Roche, Basel, Switzerland; ${ }^{3}$ Roche Nederland BV, Woerden, Netherlands

Background: Treatment with methotrexate (MTX), often with concomitant glucocorticoids, is the cornerstone of early rheumatoid arthritis (RA) therapy. However, it may be less effective compared to (expensive) biological disease modifying anti-rheumatic drugs, such as tocilizumab (TCZ). Hitherto, the effectiveness and safety of MTX in combination with glucocorticoids have never been compared to TCZ with or without MTX.

Objectives: To compare effectiveness and safety of initiating TCZ, or TCZ with MTX (TCZ+MTX) to initiation of MTX with $10 \mathrm{mg}$ prednisone (MTX+Pred) all in a step-up treat-to-target treatment strategy in early RA patients.

Methods: Individual patient data of the U-Act-Early $(n=317)$ and CAMERA-II $(n=236)$ trials were used. Both were 2-year, double-blind, randomised, placebo-controlled studies evaluating step-up tight-control, treat-totarget treatment strategies with the opportunity to taper, in case of sus tained remission, TCZ and/or MTX.1,2 Using MTX $(n=108+119)$ as the reference strategy, TCZ+MTX $(n=106)$ and TCZ $(n=103)$ were compared with $\mathrm{MTX}+$ Pred $(n=117)$ : primary outcome was the disease activity score (DAS28) over time. Secondary outcomes were remission, defined as DAS28<2.6, and the ConRew score (cumulative occurrence of remission and sustainment of remission). To assess the influence of acute phase reactants (APRs) on the results a disease activity outcome without APRs was also analysed (i.e. CDAl, modified due to lack of VAS physician in CAMERA-II). Multiple imputation was used for missing baseline data: $H A Q$, rheumatoid factor (RF) and smoking status. Multi-level models were used to account for clustering of patients within trials and for repeated measurements within patients over time. All models were corrected for baseline DAS28, HAQ, RF-status and smoking using fixed (and random) effects.

Results: Differences between U-Act-Early and CAMERA-II for RF seropositivity and DAS28 at baseline were observed; respectively $73 \%$ vs. $60 \%$ $(p<0.01)$ and 5.2 vs. $5.7 \quad(p=0.01)$. DAS28 was statistically significantly lower over time for TCZ+MTX compared to MTX+Pred (mean difference: $-0.62[95 \% \mathrm{Cl}-1.14$ to -0.10$]$ ), but not for TCZ, Table 1. Remission occurred more often in TCZ+MTX and TCZ compared to MTX+Pred: relative risk $1.11[95 \% \mathrm{Cl} 1.02$ to 1.22$]$ and 1.09 [1.00 to 1.20$]$, respectively ConRew scores were in line but not statistical significant different, Table 1. When using modified-CDAI, TCZ strategies did not show better control of disease activity over time than MTX+Pred (mean difference in log transformed values: TCZ+MTX vs. MTX+Pred: $0.10[95 \% \mathrm{Cl}-0.16$ to 0.36$]$ TCZ vs. MTX+Pred: $0.24[95 \% \mathrm{Cl}-0.04$ to 0.52$])$, Table 1 . No differences in safety outcomes could be established, Table 2 .

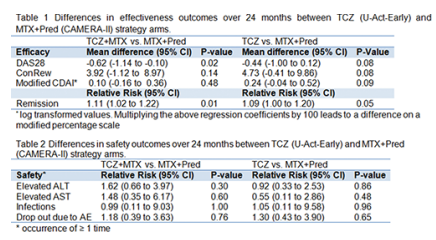

Conclusion: In early RA patients, TCZ-based strategies resulted in better DAS28 over time compared to MTX+Pred, as well as higher percentage of remission, part of these effects may be due to a specific effect of TCZ on APRs.

REFERENCES:

[1] Bijlsma JWJ, et al. Lancet. 2016;388:343-55.

[2] Bakker MF, et al. Ann Intern Med. 2012;156:329-339.

Disclosure of Interests: Maxime Verhoeven: None declared, Janneke Tekstra: None declared, Jacob M. van Laar Grant/research support from: Genentech, Consultant for: F. Hoffmann-La Roche, Johannes WJ Bijlsma Grant/research support from: The department of the author who included patients (JWJB) in the U-Act-Early trial received reimbursements from Roche Nederland BV. JWJB reported grants and fees from Roche, AbbVie, Bristol-Myers Squibb, Merck Sharp \& Dohme, Pfizer, and UCB University Medical Center Utrecht, Utrecht University, Consultant for: SUN Pharma, Speakers bureau: Lilly, Roche, Attila Pethoe-Schramm Shareholder of: F. Hoffmann-La Roche, Employee of: F. Hoffmann-La Roche Michelle Borm Employee of: An employee of Roche Nederland BV, Floris Lafeber Shareholder of: ArthroSave, Grant/research support from: FOREUM; Dutch Arthritis Society, Paco Welsing: None declared DOI: 10.1136/annrheumdis-2019-eular.3276

\section{FRI0170 PHYSICAL ACTIVITY LEVEL IN RHEUMATOID ARTHRITIS: A SYSTEMATIC REVIEW}

Charlotte Schouller ${ }^{1}$, Jean Francis Maillefert ${ }^{2}$, Jean Marie Casillas ${ }^{3}$, Daniel Wendling ${ }^{4}$, Frank Verhoeven ${ }^{4} .{ }^{1} \mathrm{CH}$ Montceau, Rheumatology, Montceau Les Mines, France; ${ }^{2} \mathrm{CHU}$ Dijon, Rheumatology, Dijon, France; ${ }^{3} \mathrm{CHU}$ Dijon, Physical rehabilitation, Dijon, France; ${ }^{4} \mathrm{CHRU}$ Besancon, Rheumatology, Besançon, France

Background: Rheumatoid arthritis (RA) is one of the most common inflammatory rheumatism characterized by an increased cardiovascula risk. Regarding the last EULAR recommendations, physical activity is an important part of the management of RA. The evaluation of Physical activity level is needed to know RA patients practices. However, to our knowledge, there is no consensual measure tool of the physical activity level for patients suffering RA.

Objectives: The aim of this study is to evaluate the different methods of measurement of physical activity levels in RA.

Methods: This is a systematic review of literature realized on the pubmed and Cochrane databases and meeting the PRISMA recommendations. We used the following key words: « physical activity ", « physical activity level " AND « rheumatoid arthritis ». We included only article written in English language and with RA patients older than 18 years old.

Results: We identified 190 studies with the key words, 51 were selected on title and 23 articles have been identified as eligible. Finally, 19 stud ies were included in this review. In total, of the 19 selected studies, 13567 RA patients were evaluated on their level of physical activity There were $73,4 \%$ female with a mean age of 56,1 years. In 10 studies the BMI was available with results between 25 and $30 \mathrm{~kg} / \mathrm{m} 2$. Two meth ods for measuring physical activity levels have been identified. 